\title{
ERRATA
}

\section{EFFECTIVE POTENTIAL FOR D-BRANE IN CONSTANT ELECTROMAGNETIC FIELD}

\author{
[INT. J. MOD. PHYS. A, Vol. 13, No. 13 (1998) 2165-2178] \\ SHIN'ICHI NOJIRI* and SERGEI D. ODINTSOV ${ }^{\dagger}$
}

In the paper, Eq. (78) is incorrect and it should be

$$
\begin{gathered}
\left\{-\frac{1}{4}\left(\hat{G}^{-1}\right)^{j k}\left(\hat{G}^{-1}\right)^{l i}+\frac{1}{8}\left(\hat{G}^{-1}\right)^{j i}\left(\hat{G}^{-1}\right)^{l k}\right\} F_{i j} F_{k l} \\
=\frac{1}{2} \hat{G}^{-1} R^{2}\left(F_{01}^{2}+F_{02}^{2}\right)+\frac{1}{2} \hat{G}^{-2} R^{4} F_{12}^{2} .
\end{gathered}
$$

Here the coefficient in the second term in the second line is corrected. Due to the above correction, Eqs. (81), (82) and (83) are modified. Especially the total one-loop potential (83) is given by

$$
V_{T}=k\left(R^{4}+h^{2}\right)^{\frac{1}{2}}+\frac{d-2}{2} \cdot \frac{R}{\left(R^{4}+h^{2}\right)^{\frac{1}{2}}} f_{T}(1,1) .
$$

Different from the result in the text, the potential is stable and has nontrivial minimum as in Eq. (19) of the text.

\footnotetext{
*E-mail: nojiri@cc.nda.ac.jp

†E-mail : odintsov@quantum.univalle.edu.co or sergei@ecm.ub.es
} 